\title{
Utilização de bioindicadores na avaliação de impacto e no monitoramento da contaminação de rios e córregos por agrotóxicos
}

\author{
Use of bioindicators for assessing and monitoring pesticides \\ contamination in streams and rivers
}

\author{
Ana Rosa Linde Arias ${ }^{1}$ \\ Daniel Forsin Buss ${ }^{2}$ \\ Carla de Alburquerque ${ }^{1}$ \\ Alan Ferreira Inácio ${ }^{1}$ \\ M arina Moreira Freire ${ }^{1}$ \\ Mariana Egler ${ }^{2}$ \\ Riccardo Mugnai 2 \\ Darcilio Fernandes Baptista ${ }^{2}$
}

'Laboratório de

Toxicologia, Centro de

Estudos da Saúde do

Trabalhador e Ecologia

Humana, Fundação

Oswaldo Cruz. Rua

Leopoldo Bulhões 1480,

Manguinhos. 21041-210

Rio de Janeiro RJ.

arlinde@ensp.fiocruz.br

¿Laboratório de Avaliação e

Promoção da Saúde

Ambiental, Departamento

de Biologia, IOC, Fiocruz.
Abstract The objective of this article is to present an analysis of themain bioindicatorsthat are currently used to assess the environmental impact of pollution in water resources. The simplequantification of chemicals in the environment is not enough to reveal the real effects of contamination on ecosystems, making necessary the assessment of the biological effects that pollution causes at different hierarchical levels. Thebioindicatorsused in this articleon two casestudies comprehend different hierarchical levels: in casestudy 1, three organization levels were utilized: individual, cellular and molecular, to detect the early effects of exposition to environmental pollutants in three hydrographic basins. By observing the inhibition of AChE activity in fish it was possible to assess the effects of organophosphorate and carbamate pesticides, showing the effects of agricultural activities. In case study 2, we present an assessment at the macroinvertebrate community level using the Extended Biotic Index. We discuss the advantages and limitations in the production of reliable data that could be used in the implementation of adequateactionsto protect and/or recover ecosystems. Key words Impact assessment, Environmental monitoring, Agrochemicals
Resumo Este artigo tem como obj etivo apresentar uma análise dos principais bi oindicadores utilizados para avaliação do impacto ambiental em recursoshídricos, já quea si mples mensuração dos níveis desubstâncias químicas presentes no ambiente não é suficiente para revelar os reais efeitos adversos da contaminação, tornando-se necessário a avaliação dos efeitos biológicos da contaminação em diversos níveis hierárquicos. Os bioindicadores foram tratados neste artigo através de dois estudos de casos que abrangem diferentes níveis; no caso 1, foram utilizados três níveis de organização: individual, celular e molecular, para detecção precoce dos efeitos reais da exposi ção de peixes aos poluentes ambientais em três bacias hidrográficas. A inibi ção da atividade da AchE em peixes possi bilitou a avaliação dos efeitos dos agrotóxicos organofosforados e carbamatos, evidenciando 0 efeito dos cultivosagrícolas; no caso 2, apresentamos uma avaliação no nível da comunidade de macroinvertebrados, empregando o Índice Biótico Estendido. Discutimos, as vantagens e limitações na produção de dados confiáveis que possibilitem a implementação de medidas adequadas para o diagnóstico em diferentes escalas, visando a proteção e/ou recuperação dos ecossistemas. Palavras-chaves Avaliação de impacto, M onitoramento ambiental, Agrotóxico 
Introdução

Nos últimos anos, o nível de compostos xenobióticos nos ecossistemas aquáticos vem aumentado de forma alarmante como resultado da atividade antropogênica sobre o meio ambiente. Tal fato tem contribuindo para a redução da qualidade ambiental, bem como para o comprometimento da saúde dos seres vivos que habitam esses ecossistemas ${ }^{1}$.

A biota aquática está constantemente exposta a um grande número de substâncias tóxicas lançadas no ambiente, oriundas de diversas fontes de emissão. A descarga de lixos tóxicos provenientes de efluentes industriais, os processos de drenagem agrícola, os derrames acidentais de lixos químicos e os esgotos domésticos lançados em rios e mares contribuem para a contaminação dos ecossistemas aquáticos com uma ampla gama de agentes tóxicos como metais pesados, agrotóxicos, compostos orgânicos, entre outros ${ }^{2}$.

Os principais contaminantes de origem agrícola são os resíduos de fertilizantes e os agrotóxicos. Esses produtos, quando aplicados sobre os campos de cultivo, podem atingir os corpos d'água diretamente, através da água da chuva e da irrigação, ou indiretamente através da percolação no solo, chegando aos lençóis freáticos. Outras formas de contaminação indireta podem ocorrer através da volatilização dos compostos aplicados nos cultivos e pela formação de poeira do solo contaminado 3 e/ou da pulverização de pesticidas, que podem ser transportados por correntes aéreas e se depositarem no solo e na água, distantes das áreas onde foram originalmente usados. Tendo em vista o potencial dispersivo desses produtos, para a melhoria da qualidade da água dos rios, se faz necessária uma abordagem que considere a bacia hidrográfica como unidade de estudo.

Os métodos analíticos tradicionais capazes de avaliar pesticidas organofosforados e carbamatos em água são realizados por cromatografia gasosa (GC), cromatografia líquida de alta performance (HPLC) ou espectofotometria de massa (MS) ${ }^{4}$. Devido aos problemas associados ao uso indiscriminado dos agrotóxicos, é fundamental que o monitoramento ambiental seja realizado constantemente. No entanto, esses métodos de análise exigem mão-de-obra especializada eapresentam alto custo operacional, o que torna inviável sua aplicação em larga escala ${ }^{5}$. Além disso, a poluição ambiental associada ao uso de pesticidas é especialmente difícil de ser avaliada, pois os eventos de contaminação obedecem a uma dinâmica espacialmente difusa e temporalmente variável. 0 resultado é um quadro de contaminação baixa e contínua, que nem sempre é captado pelos métodos de screening químicos, pois esses registram apenas 0 instante exato em que foram coletadas as amostras enão acompanham a variação temporal e espacial da contaminação.

Desde a década de 1970, pesquisadores e gestores de recursos hídricos da Europa 0 cidental e América do N orte ${ }^{6}$ argumentam que as metodologias tradicionais de classificação de águas, baseadas em características físicas, químicas e bacteriológicas, não são suficientes para atender aos usos múltiplos da água, sendo particularmente deficientes na avaliação da qualidade estética, de recreação eecológica do ambiente. Outra desvantagem é que, se as medições químicas forem feitas longe da fonte poluente, não serão capazes de detectar perturbações sutis sobre o ecossistema ${ }^{7}$. Para a realização de avaliações eficientes, édefundamental importância a aplicação de análises integradas da qualidade da água, unindo as repostas das metodologias tradicionais de avaliação aos aspectos biológicos do sistema7 7,9, Q Quando lançadas no ambiente aquático, as substâncias oriundas das atividades agrícolas são capazes de interagir com o organismo vivo, causando múltiplas alterações que podem gerar graves desequilíbrios ecológicos, dependendo do grau de contaminação edo tempo deexposição. Tradicionalmente, as técnicas para a avaliação destes impactos utilizando bioindicadores vêm sendo divididas em duas abordagens principais: aquelas associadas aos níveis superiores de organização, tais como populações, comunidades e ecossistemas ou no nível individual - que trata de alterações comportamentais, malformações, mudanças nas taxas de crescimento, reprodução, alimentação, alterações bioquímica e fisiológica - que inclui alterações na integridade da membrana celular, no transporte de íons, no metabolismo celular e em atividades enzimáticas. Os componentes dessa última abordagem são também chamados de bioindicadores, e são definidos como componentes biológicos, células, processos bioquímicos, estruturas e funções biológicas, alteradas quando em contato com compostos xenobióticos ${ }^{7}$.

Indicadores em diferentes níveis de organização biológica fornecem informações complementares, necessárias para a análise de risco ecológico. Osimpactos da contaminação por agrotóxicos em peixes e nos macroinvertebrados variam de acordo com os tipos de substâncias empregadas, com a sua toxicidade e com a estabilidadenos ambientes aquáticos. Os efeitos sobre a fauna incluem 
desdealterações fisiológicas em al guns organismos, até a morte maciça de populações, afetando toda a estrutura da comunidade ${ }^{10}$.

Em geral, quanto maior a concentração de pesticidas e mais longo o tempo de exposição, maiores as chances dos impactos negativos atingirem níveis superiores de organização biológi$\mathrm{ca}$, como comunidades e ecossistemas. Se um estresse dura tempo suficiente para levar à morte uma população de organismos, afetando as taxas de crescimento e de reprodução e impedindo o recrutamento de novas espécies, ela é então capaz de alterar a estrutura da comunidade ${ }^{6}$ (Figura 1). Os efeitos dos contaminantes em níveis de organização biológica mais baixos ( $p$. ex., respostas moleculares e bioquímicas) ocorrem mais rapidamente, além do que a especificidade das respostas e nossa compreensão sobre os efeitos dos contaminantes são geralmente maiores nos níveis mais baixos de organização. Geralmente, os efeitos em tais níveis podem ser diretamente ligados à exposição aos agentes contaminantes. Por exemplo, a presença de resíduos químicos e metabólitos éum in- dicador direto da disponibilidade de contaminantes para os organismos.

Neste trabalho, são apresentadas as aplicações de ferramentas de biomonitoramento aos efeitos do uso de pesticidas em dois níveis de organização biológica: organismo e comunidade. Neste contexto, o objetivo deste estudo é investigar as possibilidades de definir e utilizar uma "abordagem integrada" para avaliar o efeito tóxico desubstâncias poluentes em ecossistemas aquáticos, através do desenvolvimento e aplicação de uma bateria de bioindicadores com quatro níveis de complexidade (comunidade, individual, celular e molecular), e assim determinar o grau de impacto causado no ecossistema deuma determinadaárea, utilizando peixes e macroinvertebrados como indicadores de efeitos biológicos. Os conhecimentos delineados neste projeto serão essenciais para desenhar estratégias eficazes que permitam reparar ou recuperar a biodiversidade dos ecossistemas. A incorporação dos bioindicadores nos programas de vigilância e controle da contaminação ambiental do meio aquático proporcionaráum conhecimento mais preciso da qualidadeambiental.

Figura 1

Representação esquemática da ordem seqüencial de respostas a poluentes dentro de um sistema biológico.

DANO:

Ecossistema
Comunidade
População
Organismo
Sistêmico (órgão)
Tecido
Celular
Sub-celular (organela)
Molecular


Estudo de caso 1: utilizando bioindicadores

No nível deorganização biológica do organismo, as ferramentas de análise são chamadas de bioindicadores ${ }^{11}$. Bioindicadores são definidos como qualquer resposta a um contaminante ambiental ao nível individual, medidos no organismo ou matriz biológica, indicando um desvio do status normal que não pode ser detectado no organismo intacto. Ou seja, são medidas de fluidos corporais, células, tecidos ou medidas realizadas sobre o organismo completo, que indicam, em termos bioquímicos, celulares, fisiológicos, compartimentais ou energéticos, a presença de substâncias contaminantes ou a magnitude da resposta do organismo alvo ${ }^{12}$.

Existem bioindicadores moleculares, celulares eao nível do animal completo. As duas características mais importantes dos bioindicadores são: a) permitem identificar as interações que ocorrem entreos contaminantes eos organismos vivos; b) possibilitam a mensuração de efeitos sub-letais. Esta última característica permite pôr em prática ações remediadoras ou, melhor ainda, ações preventivas. Daí a importância e o interesse atual de incorporação da análise de bioindicadores em programas de avaliação da contaminação ambiental.

Os aspectos comuns entre organismos diferentes se acentuam principalmente ao nível molecular e, por isso, muitos bioindicadores moleculares possuem a vantagem de poderem ser aplicados a uma ampla variedade deorganismos vivos $^{13}$. Este tipo de bioindicador é de grande importância como sinalizador inicial da exposi ção aos contaminantes e de seus efeitos. Como exemplo deste tipo de bioindicador podemos citar a enzima acetilcolinesterase (AChE) que pode ser utilizada na avaliação da contaminação ambiental por agentes anticolinesterásicos, pois estes compostos se ligam à enzima gerando propagação contínua do impulso nervoso e inibem sua atividade. Vários são os agentes anticolinesterásicos conhecidos, sendo os mais importantes os organofosforados e carbamanto ${ }^{14}$, pesticidas largamente utilizados em atividades agrícolas e campanhas de saúde pública como "mata-mosquitos".

Ao nível celular, pode ser utilizado o teste do micronúcleo como bioindicador de genotoxicidade, uma vez que associações positivas entre exposição a misturas de pesticidas e a presença de micronúcleos tem sido observada em diversos estudos realizados com estes compostos ${ }^{15}$. Os micronúcleos são massas de cromatina citoplas- mática com o aspecto de pequenos núcleos, constituídos principalmente por fragmentos cromossômicos ou por cromossomos retardados durante a migração anafásica, que se detectam em células interfásicas mediante técnicas simples de coloração, aparecendo no citoplasma como pequenos fragmentos basófilos. Os micronúcleos podem aparecer por várias causas, entre elas por fal ha mitótica, tanto defragmentos acêntricos de cromossomos, gerados por ruptura (clastogenicidade), quanto de cromossomos completos (aneuploidia), como conseqüência, geralmente, de enfermidades genéticas.

Ao nível individual, os parâmetros mais comuns a serem levados em conta quando se estudam populações depeixessão o tamanho eo peso dos peixes, já que podem fornecer um indicativo da condição geral dos mesmos. 0 fator de condição éum índice que relaciona peso etamanho de peixes sendo, desta forma, útil para estimar o estado geral de saúde do peixe.

A análise cuidadosa de todos os parâmetros anteriormente citados possibilita avaliar se os animais estão ou não em suas condições fisiológicas normais em um específico ecossistema. Desta forma, objetivou-se com este estudo avaliar o efeito da poluição aquática causada por agrotóxicos em peixes através do uso de bioindicadores em três níveis de organização.

\section{Estudo de caso 1: material e métodos}

Para avaliar o grau de impacto, foi utilizada uma bateria de bioindicadores baseados em três níveis distintos de organização: individual, celular e molecular. Foram determinados os seguintes parâmetros:

- Ao nível individual: foi determinado o fator de condição como indicador da condição geral da saúde dos indivíduos de acordo com a fórmula: peso (g) / (comprimento padrão (cm) ) ${ }^{3} \times 100$;

- Ao nível celular: foi realizada a contagem de micronúcleos por cada mil eritrócitos. Foi utilizada a coloração de M ay Grunwald Giemsa para posterior visualização em microscópio óptico;

- Ao nível molecular: a atividade da AChE, um indicador do efeito da contaminação por pesticidas organofosforados e carbamatos, foi determinada segundo a metodologia descrita por Silva ${ }^{16}$, modificada por Albuquerque ${ }^{17}$.

O local escolhido para implementar o presente estudo foi a bacia do rio Paraíba do Sul devido a sua importância em um contexto ecológico, econômico e de Saúde Pública. Esta bate- 
ria de biondicadores foi estudada em acarás ( $\mathrm{Ge}$ ophagus brasiliensis) e em tilápias (Oreochromis niloticus). Essas espécies foram utilizadas como bioindicadores, pois apresentam uma ampla distribuição geográfica e características ecológicas e fisiológicas similares, conhecidas e favoráveis neste tipo de estudo. A tilápia éuma espécie exótica invasora enquanto o acará é autóctona destes ecossistemas, o que torna interessante comparar estas espécies na avaliação do impacto da poluição.

Entre as diferentes regiões desta bacia, foi escolhido o município de Barra Mansa ( $22^{\circ} 32^{\prime} \mathrm{S}$, $44^{\circ} 10^{\prime} \mathrm{W}$ ), queapresenta características que contribuem para a contaminação das águas fluviais por efluentes industriais. A região de Paty do Alferes ( $\left.22^{\circ} 25^{\prime} \mathrm{S}, 43^{\circ} 25^{\prime} \mathrm{W}\right)$, com características agrícolas, foi estudada com o objetivo de avaliar o efeito da contaminação ambiental por pesticidas sobre a biota do ecossistema. 0 rio Guandu $\left(22^{\circ} 48^{\prime} \mathrm{S}, 43^{\circ} 37^{\prime} \mathrm{W}\right.$ ) foi escolhido como controle e devido a sua importância por ser a fonte de abastecimento de água do município do Rio de Janeiro.

\section{Estudo de caso 1: resultados}

Espécimes de acará e tilápia foram coletadas nos locais selecionados objetivando efetuar-se uma análise integral utilizando a bateria de bioindicadores proposta. M ediante esta abordagem, foi possível observar a diferença nas respostas dos animais à poluição aquática em diferentes níveis de organização. Seguirão abaixo as descrições dos resultados específicos de cada bioindicador utilizado.

\section{Nível individual}

O fator de condição (Tabela I) foi utilizado buscando evidenciar modificações macroscópicas. Tanto em acarás como em tilápias foram verificadas diferenças significativas $(p<0,05)$ entre Guandu e Barra M ansa. Os acarás e as tilápias de Barra M ansa possuem o menor valor para fator de condição, demonstrando desta forma a condição geral destes peixes na área estudada. (Tabela 1 e 2).

\section{Nível celular}

Os micronúcleos e os núcleos bilobados foram analisados buscando os danos genotóxicos ocasionados por poluentes. Entre todas as locali-

\section{Tabela 1}

M édia do fator de condição em acarás, Geophagus brasiliensis, procedentes das regiões de amostragem. ( $\mathrm{N}$, número de indivíduos).

\begin{tabular}{lccc}
\hline & N & $\begin{array}{c}\text { Fator de } \\
\text { condição }\end{array}$ & $\begin{array}{c}\text { Desvio- } \\
\text { padrão }\end{array}$ \\
\hline Guandu & 29 & 3,60 & 0,76 \\
Barra Mansa & 31 & 2,77 & 0,94 \\
Paty do Alferes & 14 & 3,09 & 1,04 \\
\hline
\end{tabular}

\section{Tabela 2}

M édia do fator de condição em tilápias, Oreochromis niloticus, procedentes das regiões de amostragem. ( $\mathrm{N}$, número de indivíduos)

\begin{tabular}{lccc}
\hline & N & $\begin{array}{c}\text { Fator de } \\
\text { condição }\end{array}$ & $\begin{array}{c}\text { Desvio- } \\
\text { padrão }\end{array}$ \\
\hline Guandu & 65 & 2,73 & 1,11 \\
Barra Mansa & 17 & 2,43 & 0,76 \\
Paty do Alferes & 6 & 2,97 & 0,23 \\
\hline
\end{tabular}

dades, não foram observadas diferenças significativas $(p<0,05)$ na quantidade encontrada.

Nível molecular

$A$ atividade da enzima $A C h E$ no músculo dos acarásetilápias foi avaliada buscando analisar sua inibição pelos agrotóxicos organofosforados e carbamatos. Paty do Alferes e Barra M ansa apresentaram as menores atividades $(p<0,05)$ tanto para acarás como para tilápias (Figuras 2 e 3 ).

\section{Estudo de caso 1: discussão}

Acarás etilápias demonstraram ser espécies adequadas para o estudo do efeito da poluição nos ecossi stemas aquáticos dulciaquícolas avaliados.

Ao aplicar a bateria de bioindicadores, 0 índice que foi utilizado para avaliar o nível mais elevado (individual), ou seja, o fator de condição, já indicou uma diferença entre o estado de saúde dos peixes nos diferentes pontos. Os peixes com o estado mais saudável estão na região supostamente mais limpa, que utilizamos como controle. Porém, o fator de condição nos indica apenas o efeito geral da condição dos peixes, não 
Figura 2

Gráfico representando média da atividade da enzima AChE de Geophagus brasiliensis, acará, procedente das áreas de amostragem.

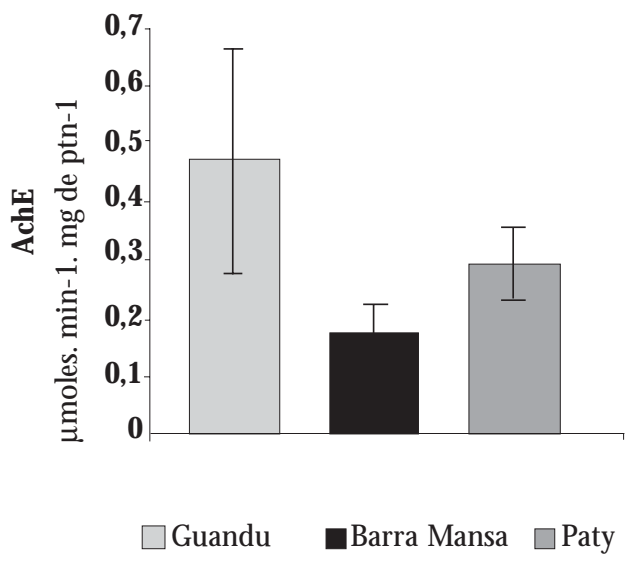

Figura 3

Gráfico representando média atividade da enzima AChE de Oreochromis niloticus, tilápias, procedentes das áreas de amostragem.

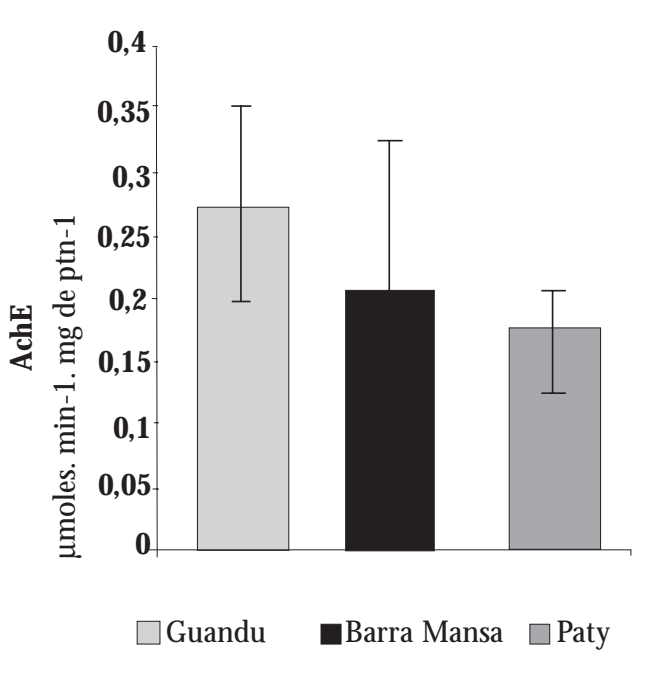

apontando como ou em que nível os peixes estariam sendo afetados pela poluição.

Descendo um nível de organização (celular), observando o bioindicador utilizado, ou seja, a presença de micronúcleos e/ou núcleos bilobados, não foram encontrados resultados que forneçam informações na avaliação dos locais estu- dados. Isto é, não foram encontrados dados que representem danos genotóxicos nos espécimens analisados, nem foram encontradas diferenças entre os pontos de amostragem.

Ao utilizarmos os indicadores mais específicos (moleculares), pôde-se ver, através da inibição da atividade da AChE, quepossibilita a avaliação do efeito dos agrotóxicos organofosforados e carbamatos na biota, que Barra M ansa e Paty do Alferes são as regiões onde os peixes estão sendo mais afetados por estes poluentes, evidenciando principalmente o efeito dos cultivos agrícolas em Paty do Alferes e mostrando que Barra M ansa, apesar de ser uma região fundamentalmente industrial, também sofre com efeitos de agrotóxicos.

Estudo de caso 2: utilizando comunidades como bioindicadores

Os indicadores biológicos são muito úteis devido a sua especificidade a certos tipos de impacto, já que inúmeras espécies são comprovadamente sensíveis a um tipo de poluente, mas tolerantes a outros ${ }^{18}$. Assim, índices podem ser criados especificamente para detectar lançamento de pesticidas, considerando as respostas de diversas espé cies da comunidade de macroinvertebrados. Os índices que têm as comunidades como unidade de estudo são eficientes para o monitoramento rápido de grandes áreas, apresentando relativo baixo custo ${ }^{19}$. Sua metodologia baseia-se em pesquisas de campo, analisando as alterações estruturais e funcionais das comunidades nos sistemas ecológicos.

Uma vez definido o objetivo do monitoramento, deve-se pensar em selecionar qual o indicador apropriado para atingi-lo. Cairns et al. ${ }^{20}$, argumenta que" basically, everything is an indicator of something but nothing is an indicator of everything". Assim, éfundamental consideramos uma série de questões antes de definirmos a estratégia a ser utilizada. Podemos começar pela escolha do grupo biológico a ser adotado. Dentre os vários grupos biológicos potencialmente úteis, temos: macrófitas, algas, peixes e macroinvertebrados, sendo estes doisúltimos os mais testados em avaliações de campo ${ }^{7}$.

A primeira abordagem visando a determinação de indicadores biológicos da qualidade das águas, com bases científicas, foi feita com bacté rias, fungos e protozoários na Alemanha por KoIkwitz \& M arsson ${ }^{21}$. Esse método ficou conhecido como índice de saprobidade e enfatizava que 
a abundância de organismos nas áreas poluídas ocorria por características fisiológicas e comportamentais que os permitia tolerar estas condições. Assim, localidades onde eram encontrados números elevados desses organismos eram classificadas como poluídas.

No final da década de 1960, iniciaram-se esforços conjuntos na Europa para testar a aplicabilidade do índice de saprobidade. Atualmente, muitos países europeus ainda utilizam metodologias baseadas na mesma filosofia, sendo chamadas de índices bióticos. Esses índices consistem em atribuir uma pontuação para cada grupo taxonômico, baseada em sua tolerância ao impacto e o somatório desses valores determina a qualidade da água do local.

Diversos índices bióticos surgiram e foram testados $5^{6,8}$ desde então. 0 objetivo deste estudo é testar a aplicação de um dos índices bióticos que vem sendo utilizados na Europa, o Índice Biótico Estendido (IBE) 22, para avaliar os efeitos da contaminação por agrotóxicos sobre a fauna de macroinvertebrados bentônicos.

\section{Estudo de caso 2: material emétodos}

Algumas considerações acerca da dinâmica de produção agrícola nortearam a elaboração do estudo. $\mathrm{N}$ a região estudada, tal qual outras bacias agriculturáveis do Estado do Rio de Janeiro, os problemas advindos da agricultura apresentam dois componentes significativos no que diz respeito ao estado de preservação dos rios: a poluição da água pelo uso de pesticidas e fertilizantes e a transformação da paisagem pelo desmatamento e aumento dos processos de erosão e assoreamento dos rios.

Sabendo que estes dois fatores agem sinergicamente sobre a biota aquática, 0 desenho experimental foi desenvolvido com o objetivo de diferenciar a resposta da comunidade presente em áreas impactadas apenas pelo desmatamento, daquela presente em áreas impactadas pelo uso de agrotóxicos. Desta forma, duas pequenas bacias hidrográficas localizadas na mesma província fisiográfica foram selecionadas para fazer parte do estudo, a microbacia do rio São Lourenço $\left(22^{\circ} 20^{\prime} \mathrm{S}, 42^{\circ} 40^{\prime} \mathrm{W}\right)$, representando uma área de agricultura intensiva, e a microbacia do rio Varginha $\left(42^{\circ} 53^{\prime} \mathrm{S}, 22^{\circ} 25^{\prime} \mathrm{W}\right.$ ), representando uma área ocupada por pastagens e casas de veraneio.

Seis trechos de rios foram selecionados para amostragens da comunidade de invertebrados aquáticos. Os pontos L1, L2, L3 e L4, localizados na bacia do rio São Lourenço, e os pontos $V 1$ eV2, na bacia do rio Varginha. Os pontos L1 eV 1 foram escolhidos para representar áreas de referência em cada uma das bacias. Os pontos L2 eV 2 referiam-se a áreas sem cobertura vegetal, ocupada por casas e pasto. Os pontos $\mathrm{L} 3 \mathrm{e}$ L4 localizavam-se ambos no rio São Lourenço, e representavam áreas de agricultura intensiva, com plantações estendendo-se até as margens dos pontos de coleta. Estes locais foram escoIhidos com basenos trabal hos de M oreira et al..$^{23}$ e Alves ${ }^{5}$, que avaliaram a presença de pesticidas anticolinesterásicos em diversos trechos do rio São Lourenço, tendo encontrado maiores concentrações de equivalentes demetil-paration nos dois pontos.

\section{Amostragem dos \\ macroinvertebrados aquáticos}

Foi feita uma amostragem quantitativa dos macroinvertebrados aquáticos com o auxílio de um amostrador do tipo Surber com malha de $125 \mu \mathrm{m}$. Três pseudo-réplicas de quatro diferentes tipos de substratos (sedimento, pedras, foIhas retidas em áreas de corenteza e folhas depositadas no fundo) foram coletadas formando um conjunto de doze amostras individuais para ser então agrupadas ecompor uma amostra para cada rio estudado.

As amostragens foram feitas em três estações do ano. No inverno (agosto, 2000); no verão (fevereiro, 2001), e no outono (maio 2001). A metodologia proposta envolveu o Índice Biótico Estendido - IBE (adaptado para a região da Serrana do Estado do Rio de Janeiro) entre localidades de referência e localidades impactadas por agricultura e desmatamento.

\section{Índice Biótico Estendido adaptado IBE-IOC}

O IBE avalia a qualidade biológica de um corpo de água baseando-se na sensibilidade de alguns grupos biológicos em relação a diversas fontes poluentes e na estimativa da riqueza total da comunidade de macroinvertebrados.

0 ÍndiceBiótico Estendido foi adaptado para a região Serrana do Estado de Rio de Janeiro, utilizando um banco de dados relativo a amostragens no período entre 1999 e 2004, em três bacias hidrográficas (Guapimirim, Grandee M acaé), com coletas realizadas em 34 pontos amostrais, quase todos coletados em três diferentes estações do ano ${ }^{24,25}$. 
Tabela 3

Tabela de cálculo do Índice Biótico Estendido adaptado para a Serra dos Órgãos no Estado do Rio de Janeiro (IBE-IOC). US - Unidades Sistemáticas.

\begin{tabular}{|c|c|c|c|c|c|c|c|c|c|c|}
\hline & & $1-10$ & $11-15$ & $16-20$ & $21-25$ & $26-30$ & $31-35$ & $36-40$ & $41-45$ & $46 \ldots$ \\
\hline PLECOPTERA, à exceção de & $>1$ US & - & - & 8 & 9 & 10 & 11 & 12 & 13 & 14 \\
\hline Paragripopteyx e Anacroneuria & 1 US & - & - & 7 & 8 & 9 & 10 & 11 & 12 & 13 \\
\hline $\begin{array}{l}\text { Paragripopteyx e } \\
\text { EPHEM EROPTERA, à exceção de }\end{array}$ & $>1$ US & - & - & 7 & 8 & 9 & 10 & 11 & 12 & \\
\hline $\begin{array}{l}\text { Baetidae, Leptohyphidae, Hylister e } \\
\text { Farrodes }\end{array}$ & 1 US & - & - & 6 & 7 & 8 & 9 & 10 & 11 & \\
\hline Anacroneuria eTRICHOPTERA, à & $>1$ US & - & 5 & 6 & 7 & 8 & 9 & 10 & 11 & \\
\hline $\begin{array}{l}\text { exceção de Calamoceratidae, } \\
\text { Glossosomatidae, H elicopsychidae, } \\
\text { Hydroptilidae, Hydropsychidae, } \\
\text { Leptoceridae }\end{array}$ & 1 US & - & 4 & 5 & 6 & 7 & 8 & 9 & 10 & \\
\hline $\begin{array}{l}\text { ISOPODA, AM PHIPODA, Hylister } \\
\text { e Farrodes }\end{array}$ & & - & 3 & 4 & 5 & 6 & 7 & 8 & 9 & \\
\hline $\begin{array}{l}\text { OLIGO CHAETA, Chironomidae, } \\
\text { Baetidae, Calamoceratidae, } \\
\text { Glossosomatidae, Helicopsychidae, } \\
\text { Hydroptilidae, Hydropsychidae e } \\
\text { Leptoceridae } \\
\text { OUTRO }\end{array}$ & & 1 & 1 & 3 & 4 & 5 & & & & \\
\hline
\end{tabular}

Tabela 4

Tabela de conversão dos valores IBE-IOC para definição das classes de qualidade da água.

\begin{tabular}{|c|c|c|c|}
\hline Valor IBESORJ & Classe de qualidade & Avaliação & Cor de referência \\
\hline$>10$ & I & $\begin{array}{l}\text { Ambiente não poluído ou não } \\
\text { alterado em maneira sensível }\end{array}$ & Azul \\
\hline $8-9$ & II & $\begin{array}{l}\text { Ambiente no qual são aparecem } \\
\text { alguns efeitos da poluição }\end{array}$ & Verde \\
\hline $6-7$ & III & Ambiente poluído & Amarelo \\
\hline $4-5$ & IV & Ambiente muito poluído & Laranja \\
\hline$<3$ & V & Ambiente fortemente poluído & Vermelho \\
\hline
\end{tabular}

\section{Estudo de caso 2: resultados}

A aplicação do Índice IBE separou as localidades em três classes de qualidade ambiental (Tabela 5).

Asáreas L1 eL2 ficaram no limiar entrea classe de referência e uma condição de impacto inicial. As áreas L3 e L4 se enquadraram em classes pobres e eram representativas de áreas de agricultura intensiva, com plantações estendendo-se até as margens dos pontos de coleta e não apresentavam qualquer contaminação por esgotos domésticos provenientes das casas dos lavradores, pois eram localizadas distantes das áreas de cultivos. Além disso, nestes locais M oreira et al. ${ }^{23}$ e Alves ${ }^{5}$ encontraram as maiores concentrações de equivalentes de metil-paration.

As áreas V 1 e V 2 foram as que apresentaram mel hores classes de qual idade da água através da análise biológica.

A fauna presente nas áreas de cultivo foi bastante afetada. Houve uma redução significativa na ocorrência de espécies e na densidade de in- 
Tabela 5

Classes de qualidade da água definidas pelo Índice Biótico Estendido - IBE para as localidades L1, L2, L3, L4 na bacia do Rio São Lourenço e localidades V 1 e V2 na bacia do rio Varginha.

\begin{tabular}{ccccccc}
\hline LOCALIDADE & OUTONO & \multicolumn{2}{l}{ INVERNO } & \multicolumn{2}{l}{ VERÃO } \\
\hline L1 & $\mathrm{I}$ & $11 / 10$ & $\mathrm{I}$ & 11 & $\mathrm{I}$ & $12 / 11$ \\
L2 & $\mathrm{I}$ & 10 & $\mathrm{I}$ & $11 / 12$ & $\mathrm{I}$ & $13 / 12$ \\
L3 & $\mathrm{IV}$ & $4 / 3$ & $\mathrm{~V}$ & 1 & $\mathrm{~V}$ & 3 \\
L4 & $\mathrm{V}$ & 3 & $\mathrm{~V}$ & 1 & $\mathrm{~V}$ & 3 \\
V1 & $\mathrm{I}$ & 11 & $\mathrm{I}$ & 10 & $\mathrm{I}$ & 10 \\
V2 & $\mathrm{I}$ & 10 & $\mathrm{I}$ & 11 & $\mathrm{I}$ & 11 \\
\hline
\end{tabular}

divíduos. Alguns táxons desapareceram como Plecoptera e Leptophlebiidae, enquanto outras ordens de insetos aquáticos foram muito reduzidas como Trichoptera, Coleopterae O donata. Nas áreas impactadas $L 3$ e $L 4$, cinco táxons responderam por $92 \%$ dos indivíduos presentes, onde os grupos dominantes foram Chironomidae, Simuliidae, Nectopsyche(Trichoptera), Americabaetis (Ephemeroptera) e Oligochaeta. Os gêneros Americabaetis e N ectopsyche ocorreram em alta densidade nos extensos bancos de algas que se desenvolveram nos locais, indicando a influência da entrada de fertilizantes.

Com relação aos locais $L 1$ e L2, à montante da área cultivada, a fauna foi constituída por grupos sensíveis como Plecoptera, Trichoptera e Ephemeroptera. Entretanto, estes ocorreram em menor densidade relativa que nas áreas $V 1 \mathrm{e}$ $\mathrm{V} 2$. Foram registrados também os maiores valores de abundância de indivíduos da ordem de Diptera (25\% em L1 e 30\% em L2). A diversidade de espécies, quando aplicado o índice de Shannon Weaver, também foi relativamente baixa $\left(H^{\prime}=1,69\right.$ em $L 1$, e $\left.H^{\prime}=1,55\right)$, confirmando os resultados do IBE.

Os locais V1 e V2, na bacia do rio Varginha, apresentaram os maiores valores quando analisados pelo IBE, sendo ambos os locais classificados como de alta integridade ambiental. Em relação à biota, estes dois pontos apresentaram a maior abundância de indivíduos da ordem Plecoptera ( $52 \%$ em V1 e $30 \%$ em V2), da família Leptophlebiidae (55\% e $21 \%$, respectivamente). Especificamente no local V1, foram registrados os maiores números de indivíduos da ordem Coleoptera (60\%) e da ordem Trichoptera (36\%), 0 que influenciou positivamente os resultados dos índices. A diversidade de espécies nos locais $V 1$ e V2 foi mais alta quando comparada com L1 eL2, sendo que o ponto $\mathrm{V} 1$ apresentou o maior valor entre todos os locais avaliados ( $H^{\prime}=2,97 \mathrm{em} \mathrm{V} 1$ e $H^{\prime}=1,93$ em V2).

\section{Estudo de caso 2: discussão}

Os resultados revelam que o índice IBE foi sensível para diferenciar as localidades quanto à qualidade da água e sua influência sobre a biota. As áreas de agricultura foram claramente separadas dos outros locais de coleta, ao passo que o índice também foi capaz de discriminar entrecondições preservadas e áreas com al guma perturbação.

A pontuação aferida aos grupos taxonômicos selecionados para comporem o índice IBE se revelaram sensíveis para identificar sinais discretos de perturbação na comunidade, que não foram percebidas pela avaliação da fisiográfica da pai sagem e uso do solo. Embora os locais L1 1 VV1 e L2 e V2 parecessem ser semelhantes enquanto áreas de referência e áreas de pasto, a aplicação do índice indicou que, nos locais L1 e L2, as populações estavam mais comprometidas que na bacia do rio Varginha.

A explicação para esse fato parece estar na influência da ocupação agrícola intensiva na bacia do rio São Lourenço. 0 uso de agrotóxicos é um risco para os invertebrados que vivem nos ambientes dulciaquícolas adjacentes as áreas de cultivo, uma vez que eles tem sistemas biológicos similares aos dos organismos alvos, podendo ser diretamente afetados pela ação inespecífica destes compostos ${ }^{26}$. 0 pool de espécies na bacia do rio São Lourenço parece estar sofrendo o impacto da aplicação de pestici das na área da bacia, refletido na diversidade e dinâmica das populações nas áreas $L 1$ e L2. A redução na densidade de populações pode ser tanto um efeito direto da 
contaminação da água, quanto um resultado indireto relacionado às mudanças nas interações entre espécies, à redução das taxas de emergência e reprodução, e à menor capacidade de dispersão e colonização na bacia.

Os resultados encontrados na bacia do rio Varginha revelaram ainda que o índice biótico aplicado foi adequado para avaliação da qualidade da água em escala de bacia. Os dois locais tinham condições ambientais bastante distintas, com o local V2, apresentando um maior comprometimento dos habitats aquáticos pelo grave assoreamento do leito e perda da vegetação marginal. Entretanto, sem o comprometimento da qualidade da água, o pool de espécies presentes foi mais diverso e eqüitativo, aumentando a performance biológica dos índices.

0 índice IBE demonstrou sua aplicabilidade na avaliação da qualidade da água em áreas afetadas pela agricultura, principalmente quanto sua sensibilidade em distinguir áreas intermediárias daquelas mais preservadas. A aplicação do IBE em outras áreas e em situações distintas de uso do solo e produção agrícola éum próximo passo para a proposição e difusão da metodologia biológica avaliada no diagnóstico ambiental de bacias agrícolas.

\section{Utilização de bioindicadores em rios e córregos}

Atualmente, o objetivo dos estudos relacionados à avaliação dos impactos em áreas agriculturáveis é o desenvolvimento e aperfeiçoamento de metodologias e ferramentas que permitam determinar de forma realista e exeqüível a condição ambiental. Além disso, deve considerar a importância do tratamento e transmissão da informação a diferentes públicos, muitas vezes leigos. Portanto, um dos desafios é tornar os dados científicos mais facilmente utilizáveis por técnicos, gestores, cientistas, políticos, grupos de interesse (associações não governamentais, cooperativas) e pelo público em geral.

0 emprego de uma bateria de bioindicadores abrangendo distintos níveis de organização biológica possibilita o conhecimento sobrea que nível o poluente interage com o organismo, e a que nível este é mais suscetível à ação daquele. Os resultados de trabal hos como este são essenciais para desenhar estratégias eficazes que per- mitam recuperar a biodiversidade dos ecossistemas aquáticos. A incorporação de Programas de Vigilância e Controle da contaminação ambiental do meio aquático através de uma bateria de bioindicadores proporcionará um conhecimento mais preciso da qualidade ambiental do mesmo (uma vez que permitirá estabelecer 0 efeito tóxico real que a contaminação exerce sobre as populações). Isto permitirá avaliar e caracterizar o estado de saúde ambiental dos ecossistemas aquáticos, produzindo dados confiáveis que possibilitarão a implementação de medidas adequadas para sua proteção e/ou recuperação, além de permitir a avaliação dos riscos que tais contaminações conferem à Saúde Pública e na biodiversidade desses ecossistemas.

Uma vez que é improvável que respostas em um único nível de organização biológica satisfaçam os critérios de especificidade, os mecanismos de compreensão e a relevância ecológica, uma abordagem alternativa é estudar respostas em diferentes níveis de organização simultaneamente, integrando os efeitos dos contaminantes através dos diferentes níveis deorganização; portanto, é necessário promover pesquisas interdisciplinares.

Concluímos que a utilização desta bateria de bioindicadores nos permitiu avaliar e formular um retrato detal hado dos diferentes efeitos das diferentes formas de poluição nos organismos aquáticos, sendo possível diferenciar o efeito dos agrotóxicos.

Os ecossistemas aquáticos de rios, em função de sua grande heterogeneidade física, que incluem de pequenos a grandes rios, variação no regime de vazão, geomorfologia do canal entre outras características do habitat. Assim, consideramos, por exemplo, que o uso da bateria de bioindicadores utilizando a comunidade de peixes seja uma metodologia mais adequada para grandes rios, pois estes apresentam, em geral, misturas complexas de poluentes; portanto, a alta especificidade de respostas dos bioindicadores torna-se mais eficiente. Além disso, a coleta de macroinvertebrados em grandes rios é muito trabal hosa e pouco eficiente. Já em rios de pequena dimensão, a fauna de macroinvertebrados pode ser extremamente rica, enquanto que a fauna de peixes pode não ser suportada, e em geral seus representantes são de pequeno porte, o que dificulta os testes com os bioindicadores disponíveis. 


\section{Colaboradores}

AR Linde-Arias e DF Baptista participaram no levantamento e análise dos dados de campo, na concepção teórica, elaboração, revisão e redação final do texto. DF Buss, C Alburquerque, AF Inácio, M M Freire, M Egler eR Mugnai participaram do levantamento e análise dos dados de campo.

\section{Agradecimentos}

Este trabalho recebeu apoio dos programas $\mathrm{Pa}$ pes III (VPPDT/FIOCRUZ) e Programa Institucional de Pesquisa em Saúde e Ambiente da FIOCRUZ (VPSRA/FIOCRUZ).

\section{Referências}

1. Cajaraville M P, Bebianno JM, Blasco J, Porte C, Sarasquete $C$, Viarengo $A$. The use of biomarkers to assess the impact of pollution in coastal environments of the Iberian Peninsula: a practical approach. Sci Total Environ 2000; 247:295-311.

2. Rashed M N. M onitoring of environmental heavy metals in fish from Nasser Lake, Environ Int 2001; 27(1): 27-33.

3. Cooper CM. Biological effects of agriculturally derived surface-water pollutants on aquatic systems - a review. J Environ Qual 1993; 22: 402-408.

4. Lacorte $S$, Barcelo D. Determination of organophosphorus pesticides and their transformation products in river water by automated on-line solid-phase extraction followed by thermospray liquid chromatography-mass spectrometry. J. Chromatogr 1995; A712: 103-112.

5. Alves SR. Avaliação dos resíduos de pesticidas organofosforados e carbamatos por metodologia enzimática no Córrego de São Lourenço, N ova Friburgo-R] [dissertação]. Escola Nacional de Saúde Pública. Fundação Oswaldo Cruz; p.61.

6. Cairns JJr, Pratt JR. A history of biological monitoring using benthic macroinvertebrates. In: Rosenberg DM, Resh VH. Freshwater biomonitoring and benthic macroinvertebrates. N ew York: Chapman \& Hall; 1993. p. 10-27.

7. Rosenberg DM, Resh VH, organizadores. Freshwater Biomonitoring and benthic macroinvertebrates. NewYork: Chapman \& Hall; 1993.

8. Metcalfe JL. Biological water quality assessment of running waters based on macroinvertebrates communities: history and present status in Europe. Environ. Pollut. 1989; 60:101-139.
9. Buss DF, Baptista DF, Nessimian JL. Bases conceituais para a aplicação de biomonitoramento em programas de avaliação da qualidade da água de rios. Cad Saúde Pública 2003; 19(2): 465-473.

10. Clements WH. Integrating effects of contaminats across levels of biological organization. J. Ecosy Stress and Rec 2000; 7:113-116.

11. Hyne RV, M aher WA. M acroinvertebrate Biomarkers: Links to Toxicosis and Changes in Population or Communities. Cooperative Centre for Freshwater Ecology. University of Canberra. Australia. Cooperative Research Centre for Freshwater Ecology. Technical Report ScD5, 2000. Disponível em: http:// enterprise.canberra. edu.au

12. Livingstone DR. Biotechnology and pollution monitoring: Use of molecular biomarkers in the aquatic environment. J. Chem. Tech. Biotechnol 1993; 57: 195-211.

13. Lama PKS, GrayJS. The use of biomarkers in environmental monitoring programmes. M ar Pollut Bull 2003. 46(2): 182-186.

14. Lionetto M G, Caricato R, Giordano M E, Pascariello $M F, M$ arinosci L, Schettino T. Integrated use of biomarkers (acetylcholinesterase and antioxidant enzymes activities) in M ytilus gallo provincialis and Mullus barbatus in an Italian coastal marine area. M ar Pollut Bull 2003; 46:324-330.

15. Bolognesi C. Genotoxicity of pesticides: a review of human biomonitoring studies. Mutat Res/Reviews in Mutat Res 2003; 543: 251-272.

16. Silva JJ, Alves SR, Inácio AF, M eyer A, Sarcinelli PN, Ferreira M F, CunhaJ C, M oreiraJC. Cholinesteraseactivities determination in frozen blood samples: an improvement to the occupational monitoring in developing countries. Hum Exp Toxicol 2000; 19(3): 173-177. 
17. Albuquerque C. Uso da acetilcolinesterase em tilápias e acarás como bioindicador de exposição a pesticidas organofosforados em ecossistemas aquáticos [ monografia de graduação]. Rio de Janeiro (RJ): Universidade Veiga de Almeida; 2004.

18. Washington HG. Diversity, biotic and similarity indices. A review with special relevance to aquatic ecosystems. Water Res 1984; 18:653-694.

19. Watzin MC, M acintosh AW. Aquatic ecosystems in agricultural landscapes: A review of ecological indicators and achievable ecological outcomes. J Soil Water Conserv 1999; 4: 636-644.

20. Cairns JJr, M cCormick PV, Niederlehner BR. A proposal framework for developing indicators of ecosystem health. Hydrobiologia 1993; 263: 1-44.

21. Kolkwitz R, M arsson M. Oekologie der tierischen Saprobien. Internationale Revue der gesamten. Hydrobiologie und Hydrographie 1909; 2:126-152.

22. Ghetti PF.I macroinvertebrati nell'analisi di qualitá dei corsi d'acqua. Manuale di applicazione. Volume allegato agli atti del convegno "esperienze e confronti nell'applicazione degli indici biotici in corsi d'acqua italiani". Ed. Provincia di Trento; 1986.
23. M oreira JC, Castro Faria M V, Jacob SC, Lima S, Alves SR, Oliveira-Silva JJ, et al. Avaliação integrada do impacto do uso de agrotóxicos sobre a saúde humana de uma comunidade agrícola de Nova Friburgo, RJ. Rev. Saúde Coletiva 2002; 7(2): 299-311.

24. M ugnai R, Carvalho AL, Oliveira RB, Sanfins CS, Baptista DF. Adaptação do Índice Biológico Extendido (IBE) para o estado do Rio de Janeiro. [CD-ROM ]. Anais do X Congresso Brasileiro de Limnologia; 2005.

25. M ugnai R, Carvalho AL, Oliveira RB, Sanfins CS, Baptista DF. Teste de sensibilidade do Índice IBE-SORJ. Índice Biológico Estendido- Serra dos Ó rgãos - Rio de Janeiro [CD-ROM ]. Congresso Brasileiro de Ecologia; 2005.

26. M aund SJ, Sherratt TN, Stickland T. Ecological considerations in pesticide risk assessment for aquatic ecosystems. Pestic. Sci 1997; 47: 185-190.

Artigo apresentado em 20/01/2006

Aprovado em 16/06/2006

Versão final apresentada em 31/08/2006 